\title{
Mitigating optical singularities in coordinate-based metamaterial waveguides
}

\author{
Sophie Viaene, ${ }^{1,2}$ Vincent Ginis, ${ }^{1}$ Jan Danckaert, ${ }^{1}$ and Philippe Tassin ${ }^{2,1, *}$ \\ ${ }^{1}$ Applied Physics Research Group, Vrije Universiteit Brussel, Pleinlaan 2, B-1050 Brussels, Belgium \\ ${ }^{2}$ Department of Physics, Chalmers University of Technology, SE-412 96 Göteborg, Sweden
}

(Received 16 December 2016; published 10 April 2017)

\begin{abstract}
Transformation optics has revolutionized our approach to material design in several scientific disciplines by determining the material properties that implement the desired effects of a coordinate transformation. Unfortunately, the performance of several coordinate-based devices, such as beam splitters and invisibility cloaks, suffers from the necessary implementation of singularities with extreme material parameters. Here, we make use of transformation optics to eliminate these singularities in an isotropic way for the improvement of coordinate-based metamaterial waveguides. In particular, singularities that lead to vanishing material properties are softened with a global rescaling of the coordinates, while singular terms that lead to infinite material properties are strategically replaced by well-behaved curve factors. Detailed full-wave simulations confirm that the resulting waveguide devices are as efficient as their singular counterparts despite the fact that they consist of materials with much more moderate optical properties.
\end{abstract}

DOI: 10.1103/PhysRevB.95.155412

\section{INTRODUCTION}

In the past decade, coordinate transformations [1-5] have been a driving force for the design of various extraordinary metamaterial devices [6-11]. These devices are engineered at the subwavelength level to materialize the desired effects of a coordinate transformation on simple material systems, reproducing the transformed physical equations with tailored macroscopic properties. In particular, the reproduction of the transformed Maxwell equations with permittivity and permeability distributions following from the framework of transformation optics $[3,4,12]$ has resulted in an impressive control of free-space light propagation [13,14], light emission [15-17], diffuse light propagation [18], nonreciprocal devices [19], nonlocal electromagnetic effects [20,21], and temporal phenomena [22-26].

Unfortunately, several interesting coordinate-based designs of beam splitters [13,14,27] and invisibility cloaks [3,4,28] rely on singularities to achieve their functionality. These singularities result in extremely high or low material properties, which must be implemented with resonant metamaterials that are often lossy and have low bandwidth [11,29-31]. Several research groups have tried to reduce these undesired effects by developing low-loss dielectric, active, and/or layered metamaterials [11,30-36] and by developing optimized quasiconformal coordinate transformations [14,37-39] or polarization-specific transformations [27,40,41]. Excitingly, transformation optics itself has also provided a valuable contribution [42-44]. By making use of an additional coordinate transformation, harmful material singularities may be transmuted into harmless geometrical singularities that can be implemented with anisotropic metamaterials with finite material parameters. This technique is very successful as long as the initial refractive index profile behaves as a power law $r^{p}$ with $p>-1$ close to the singularity and has been used to improve the implementation of an Eaton lens [45].

In this paper, we make use of transformation optics to enhance the performance of metamaterial slab waveguides

${ }^{*}$ Corresponding author. that are based on coordinate transformation with singularities, avoiding waveguide designs with vanishing thickness or infinite anisotropies in the core. In recent years, the concepts of transformation optics have been used to manipulate light that is confined to metal-dielectric interfaces [41,46-49], graphene-dielectric interfaces [50,51], and dielectric waveguides [27,52,53]. In particular, the efficient reproduction of transformed graphene-dielectric interfaces and dielectric waveguides requires an alternative materialization of the coordinate transformation, based on coordinate-dependent surface conductivities of graphene sheets [50] or thickness variations of waveguide cores [27].

Unfortunately, the aforementioned transmutation of singularities with anisotropic materials [42] cannot be used for the improvement of metamaterial waveguides, as it would invalidate the coordinate-based thickness variation [27] (see Supplemental Material, Sec. SII [54]). Instead, our main strategy is to eliminate singular contributions to the coordinate transformation by strategically replacing them with wellbehaved curve factors. We will do so for the important case of Schwarz-Christoffel transformations [55-57] that map a straight line onto a polygon with singular power maps at the vertices (Fig. 2). The replacement of these singular power maps with curve factors works very well for singularities that lead to infinite anisotropies inside the waveguide core. However, this approach does not always prevent the thickness of the waveguide from vanishing. Therefore, after a brief introduction to the design of Schwarz-Christoffel waveguides (Sec. II), this paper deals with singularities in a binary way. Singularities that lead to vanishing waveguide thickness will be softened by appropriate global rescalings (Sec. III). We will refer to these singularities as singularities of the first kind. Singularities that lead to infinite anisotropies of the core will be eliminated with curve factors (Sec. IV). We refer to these singularities as singularities of the second kind. In this way, the detrimental effects of singularities are alleviated without affecting the degree of isotropy of the design. The combination of both techniques will lead to designs with much more moderate material properties and allows guiding the waves along boundaries of any shape. 
(a)

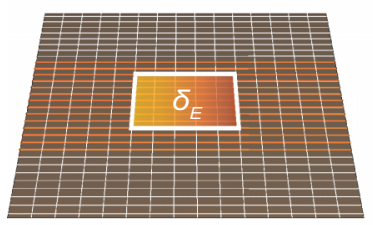

$(u, v)$

(d)

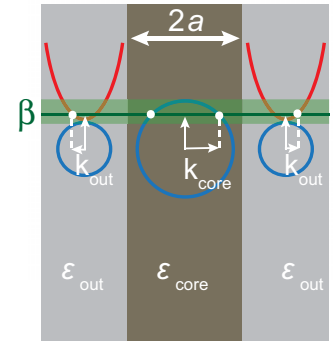

(b)

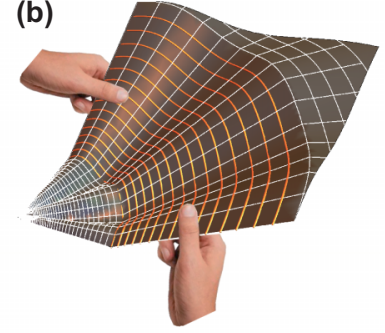

(e)

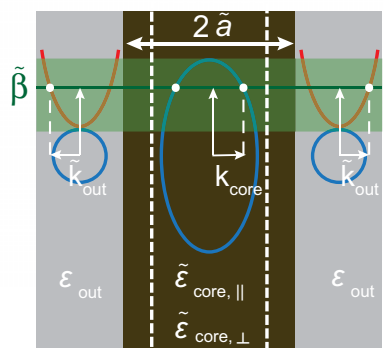

(c)

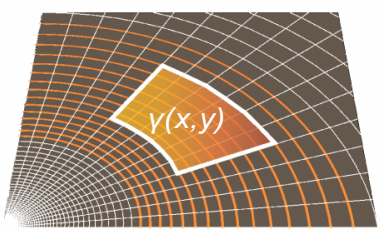

$(x, y)$

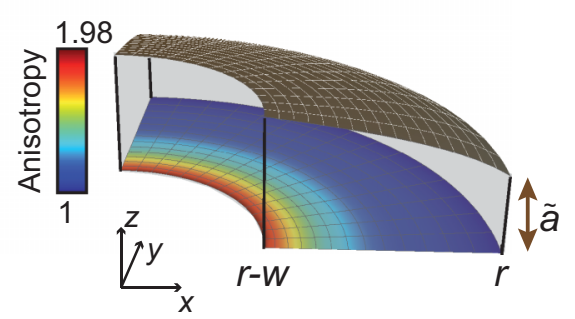

FIG. 1. Materialization of coordinate-transformed guided waves with a uniaxial waveguide core $\left(\tilde{\varepsilon}_{\| \mid}, \tilde{\varepsilon}_{\perp}\right)$ and a thickness variation $\tilde{a}$ based on Ref. [27]. (a)-(c) A two-dimensional conformal transformation transforms (a) straight light flows (orange lines) along a trivial dielectric slab waveguide into (c) curved ones by inducing a geometry component $\gamma(x, y)$ on the waveguide plane. (d) The initial guided mode along a slab waveguide with thickness $2 a$ and permittivities $\varepsilon_{\text {core }}\left(\varepsilon_{\text {out }}\right)$ in the core (outer regions) is fully characterized by an in-plane propagation constant $\beta$ (green line) parallel to the waveguide plane at a particular angular frequency $\omega$. Indeed, it determines the characteristic variations of the transverse profile, i.e., the sinusoidal variations inside the core $\left[\cos \left(k_{\text {core }} z\right)\right]$ correspond to the intersection of the green line with the blue ellipse, while the exponentially decaying fields outside of the core $\left[\exp \left(-\left|k_{\text {out }} z\right|\right)\right]$ correspond to the intersection of the green line with the red hyperbola. (e) The coordinate transformation stretches the in-plane propagation constant $\tilde{\beta}$ along the waveguide plane, which is implemented by a uniaxial material inside the core $\left(\tilde{\varepsilon}_{\|}, \tilde{\varepsilon}_{\perp}\right)$. To ensure that boundary conditions are compatible with $\tilde{\beta}$, the thickness $\tilde{a}$ has to change according to Eq. (1). (f) A visualization of the beam bender design in Ref. [27] shows that the anisotropy of the core is modest and highest at the inner radius of the bend (surface coloring on the waveguide symmetry plane). The thickness variation $\tilde{a}$ is visualized by the gray floating surface, representing the upper interface of the waveguide. Subsets of this figure have been reproduced from Ref. [27].

\section{SINGULARITIES IN METAMATERIAL WAVEGUIDES}

In recent work [27], we have reproduced desired coordinatetransformed flows of guided modes with specific metamaterial waveguides based on conformal transformations [Figs. 1(a)-1(c)]. Conformal transformations have the particular property that they preserve the angles between any two coordinate lines before and after the transformation [14,56-58]. As a result, the effect of a conformal coordinate transformation is represented by a single scalar geometry component $\gamma(x, y)$, which can be interpreted as a local stretching of the propagation constant along the waveguide $\beta \rightarrow \tilde{\beta}=\gamma(x, y) \beta$ [Figs. 1(d) and 1(e)].

To implement the coordinate stretching, we have emulated the effects of $\gamma(x, y)$ on the characteristic equations of a guided mode: The Helmholtz wave equation-describing the propagation along the waveguide-and the dispersion relation-imposing continuous fields along the interfaces $z=$ $\pm \tilde{a}$ of the waveguide. In Fig. 1(d), we consider an incident guided mode with angular frequency $\omega$ and a propagation constant $\beta$ parallel to the waveguide, whose transverse profile varies sinusoidally inside the core $\left[\cos \left(k_{\text {core }} z\right)\right]$ and decays exponentially outside of the core $\left[\exp \left(-\left|k_{\text {out }} z\right|\right)\right]$. The use of uniaxial metamaterials inside that waveguide core, with principal components $\tilde{\epsilon}_{\|}=\varepsilon_{\text {core }}, \tilde{\varepsilon}_{\perp}=\gamma(x, y) \varepsilon_{\text {core }}$ parallel and perpendicular to the waveguide plane, reproduces the transformed Helmholtz wave equation exactly. However, the initial thickness $a$ is now incompatible with the new extinction coefficient of the fields outside the core, $\tilde{k}_{\text {out }}^{2}=\gamma(x, y) \beta^{2}-$ $\varepsilon_{\text {out }} \frac{\omega^{2}}{c^{2}}$. A thickness variation $\tilde{a}(x, y)$ restores the continuity of the electromagnetic fields,

$$
\tilde{a}(x, y)=\frac{1}{k_{\text {core }}} \operatorname{atan}\left(\frac{\varepsilon_{\text {core }}}{\varepsilon_{\text {out }}} \frac{\sqrt{\gamma(\mathrm{x}, \mathrm{y}) \beta^{2}-\varepsilon_{\text {out }} \frac{\omega^{2}}{\mathrm{c}^{2}}}}{\mathrm{k}_{\text {core }}}\right) .
$$

In other words, the thickness variation ensures that the exponential tails of the mode are allowed to move at the same phase velocity as the fields inside the waveguide. Note that it is only valid in the adiabatic approximation, i.e., when the electromagnetic field resembles a local plane wave at each point $(x, y)$ and when the interfaces are approximately parallel [Fig. 1(e)] [27]. It is also important to point out (see also Supplemental Material, Sec. SII [54]) that a unique thickness variation does not exist when the in-plane components of the permittivity tensor are anisotropic. For this reason, optical singularities cannot be transmuted as proposed in Ref. [42].

The beam bender design in Fig. 1(f) does not suffer from the presence of singularities and has core anisotropies $\frac{\tilde{\epsilon}_{\perp}}{\tilde{\epsilon}_{\|}}=$ $\gamma(x, y)$ and waveguide thicknesses that are nonzero and well behaved. On the other hand, the efficient implementation of Schwarz-Christoffel waveguides will provide quite a challenge due to the appearance of isolated singularities at the vertex of a polygon [56]. As shown in Fig. 2, Schwarz-Christoffel transformations map polygonal boundaries in the $z$ plane 
(a) w-space

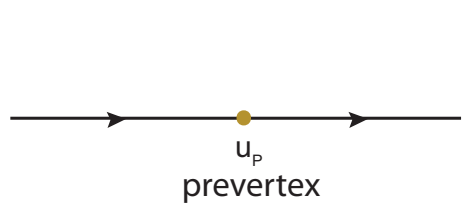

(b)

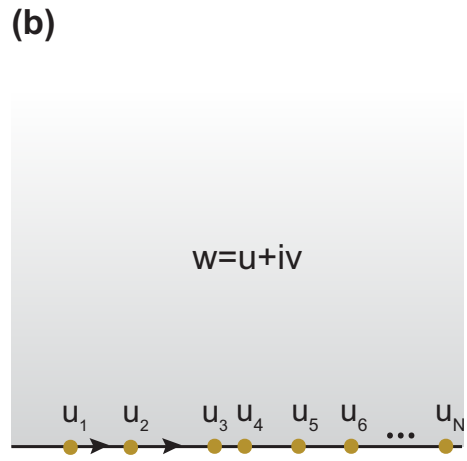

z-space

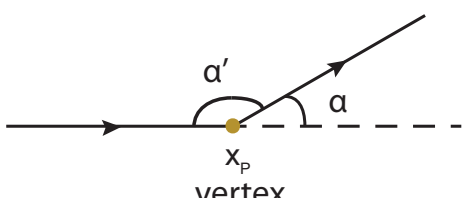

transformation

$$
d z=\frac{1}{\left(w-u_{p}\right)^{\alpha / \pi}} d w
$$

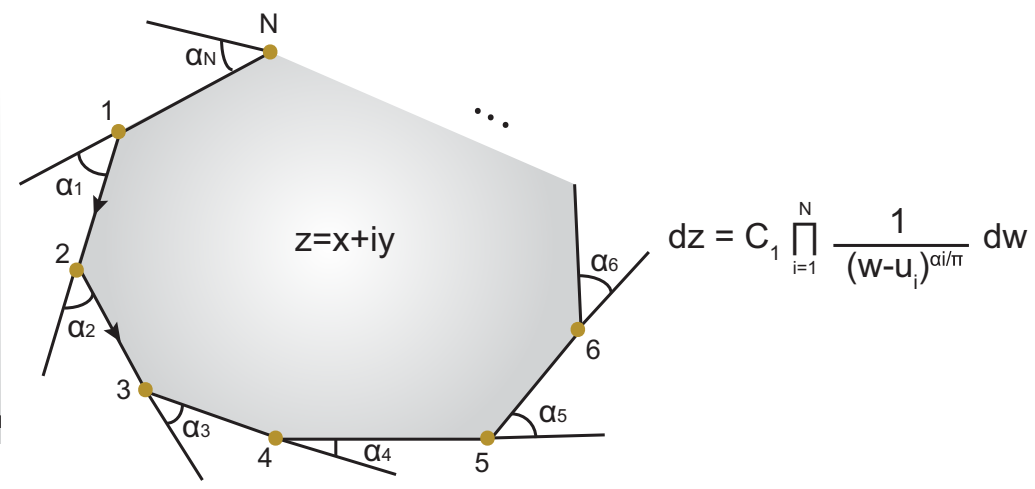

FIG. 2. A Schwarz-Christoffel transformation generates (a) vertices or (b) polygonal boundaries with a series of power maps between the complex $w$ and $z$ plane. This is achieved by making use of local power maps with exponents $1-\alpha / \pi$ (right-hand side).

onto straight boundaries in the $w$ plane by making use of a sequence of local power maps (Fig. 2). Each local power map generates a particular corner of the boundary in the $z$ plane. For example, a power map $z(w)=\left(w-u_{P}\right)^{\frac{\alpha^{\prime}}{\pi}}$ in the vicinity of a prevertex $u_{P}$ generates a sector with outer angle $\alpha=\pi-\alpha^{\prime}$. As a result, a particular polygonal boundary with outer angles $\alpha_{i}$ at $N$ isolated prevertices $u_{i}$ is generated by the following transformation rule:

$$
d z=C_{1} \Pi_{i=1}^{N} \frac{1}{\left(w-u_{i}\right)^{\frac{\alpha_{i}}{\pi}}} d w .
$$

The integral $z(w)=\int_{w_{0}}^{w} \frac{d z}{d w} d w+C_{2}$ is determined by the prevertex locations $u_{i}$ for $i$ from 1 to $N$ and the integration constants $C_{1}$ and $C_{2}$ that lead to a desired shape and orientation of the boundary with respect to an arbitrary reference point $w_{0}$ (see Supplemental Material, Sec. SI [54]). It is clear that the geometry component obtains singular values at locations corresponding to the prevertices $u_{i}$,

$$
\gamma(x[u, v], y[u, v])=\left|\frac{1}{C_{1} \Pi_{i=1}^{N} \frac{1}{\left(w-u_{i}\right)^{\frac{\alpha_{i}}{\pi}}}}\right|^{2} .
$$

These singularities lead either to vanishing thickness of the waveguide, i.e., singularities of the first kind with $\gamma(x, y) \rightarrow 0$, or to singularities of the second kind associated to infinitely high anisotropy, $\frac{\tilde{\epsilon}_{\perp}}{\tilde{\epsilon}_{\|}}=\gamma(x, y) \rightarrow \infty$.

\section{A SINGLE POWER MAP}

We consider how a single power map $w=R z^{2}$ with a singularity of the first kind at the origin of the $z$ plane splits a guided wave [Fig. 3(a)],

$$
\begin{aligned}
& u=R\left(x^{2}-y^{2}\right) \\
& v=R 2 x y
\end{aligned}
$$

in a way that depends on a normalization parameter $R$. The thickness of the waveguide [Eq. (1)] vanishes when the inplane geometry,

$$
\gamma(x, y)=4 R^{2}\left(x^{2}+y^{2}\right)
$$

reaches the critical value $\gamma_{c}=\epsilon_{\text {out }} \omega^{2} / \beta^{2} c^{2}$ on a circle surrounding the singularity. Close to and within the circle, the transformation must be implemented in an approximate way. For example, one might truncate the transformation inside a disk of larger radius $r_{L}=\sqrt{\gamma_{L}} / 2 R$ so that the waveguide thickness obtains a small value $a_{\min }$ and the anisotropy of the core $\gamma_{L}$ is finite. As long as the truncated region lies sufficiently close to the singularity, it is not expected to have a profound impact on the performance of the device. Indeed, close to the singularity, the local wavelength becomes very large and the wave becomes insensitive to small perturbations. Note that a truncation would not resolve the issue for a singularity of the second kind for which the local wavelength becomes very small and the waves would be very sensitive to implementation errors.

The black circles in Fig. 3(a) represent several truncation disks, each corresponding to a particular combination of a minimal thickness $a_{\min } \in\{1.3 \cdots 6.7\} 10^{-2} \lambda_{0}$ and a normalization parameter $R \in\{1,2,3,4\}$ for guided waves with an incident free-space wavelength of $1.5 \mu \mathrm{m}$ and an initial waveguide thickness of $2 a=0.4 \mu \mathrm{m}$. As shown in Fig. 3(a), the radii of the truncation disks barely depend on $a_{\min }$ - which is deeply subwavelength - and vary strongly with $R$. Therefore, it is expected that the performance of the waveguide splitter will mainly improve with $R$. As $R$ increases, the truncations disks become smaller, so the transformation is more accurately represented close to the singularity.

This hypothesis is confirmed by full-wave numerical simulations of waveguides that implement truncated power maps for the aforementioned combinations of $R$ and $a_{\min }$. From these 
(a)

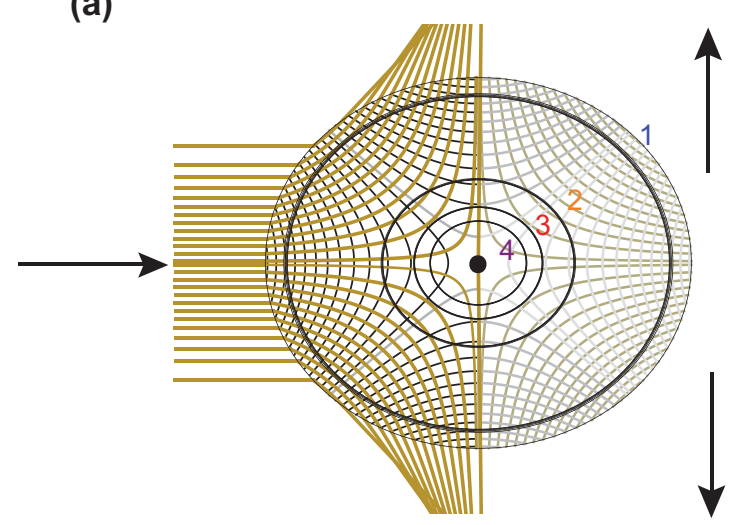

(c)

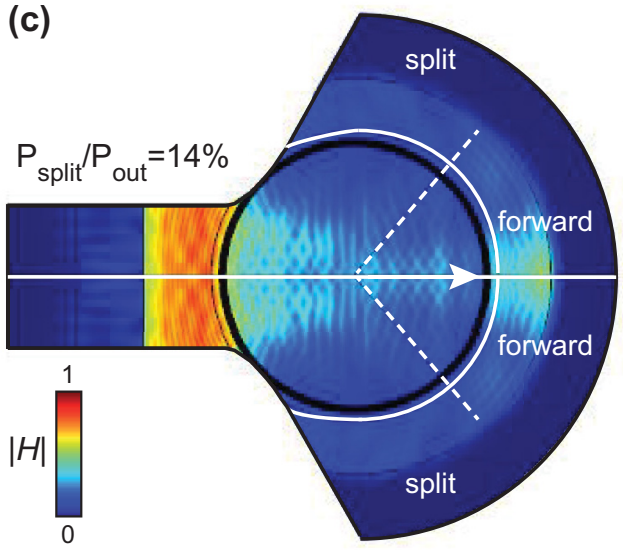

(b)

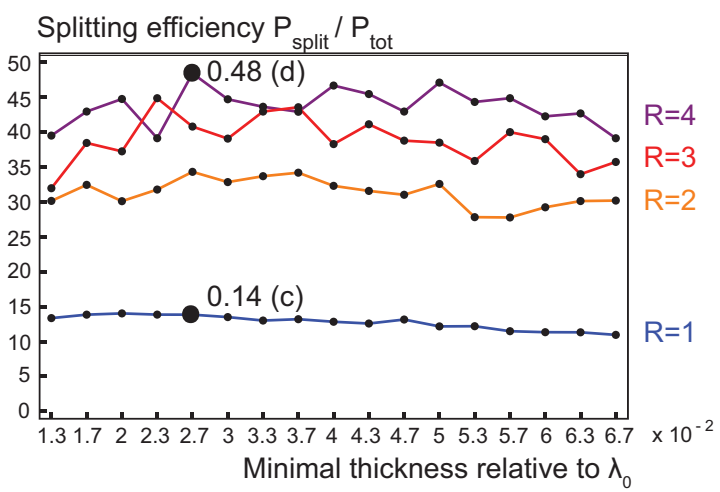

(d)

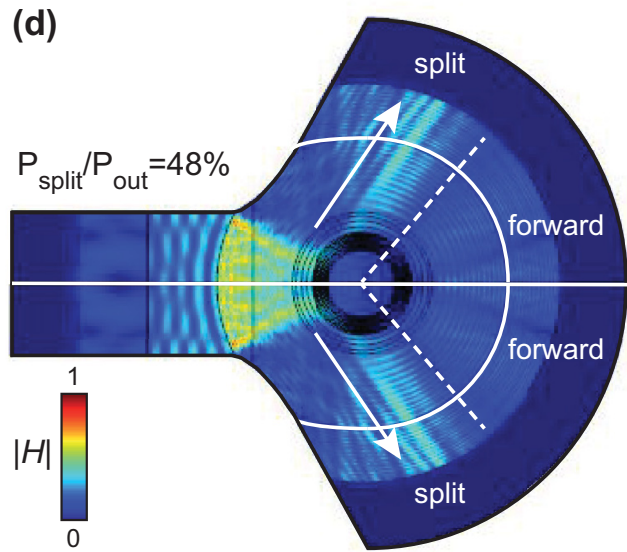

FIG. 3. The effects of a singularity of the first kind on the performance of a metamaterial waveguide, based on a power map $w=R z^{2}$, for a guided mode corresponding to a free-space wavelength of $1.5 \mu \mathrm{m}$ and initial waveguide thickness of $2 a=0.4 \mu \mathrm{m}$. (a) Light-ray trajectories, obtained from transformation optics, split when approaching the singularity. Truncations impose constant minimal anisotropies within the black circles corresponding to different scaling parameters $R$ and minimal thicknesses $a_{\min }$. (b) The efficiency, defined in terms of the power $P_{\text {split }}$ that is split and the total power $P_{\text {tot }}$ that comes out of the waveguide, largely depends on the scaling parameter $R$, while it is almost insensitive to the minimal thickness. (c) When $R$ is equal to one, the norm of the magnetic field coming from the left does not change direction as the embedded transformation in the white circle is almost entirely truncated (disk within the gray-dashed circle). (d) With a large scaling $(R=4)$, the magnetic norm splits efficiently because the transformation is implemented in regions closer to the singularity. It is only truncated inside the gray-dashed circle with a reduced radius. The power flow, which is proportional to the square of the magnetic norm, is highlighted by the white arrows.

simulations, we may evaluate the performance of the device in terms of its splitting efficiency $P_{\text {split }} / P_{\text {out }}$, i.e., the ratio between the power that is split $P_{\text {split }}$, both upwards and downwards, and the total power $P_{\text {out }}$ that emerges from the waveguide [Fig. 3(b)]. Qualitatively, the magnetic norm in Figs. 3(c) and 3 (d) confirms that high normalization parameters $R$ reduce the adverse effects due to the singularity. For small normalization parameters $(R=1$ with splitting efficiency of $14 \%)$, the guided waves barely split, while for high renormalization parameters $(R=4$ with splitting efficiency of $48 \%)$, the guided waves split efficiently. Figure 3(b) shows that the splitting efficiency increases considerably with $R$ and stays approximately constant with the truncation thickness $a_{\min }$, thereby confirming the previous observations. Therefore, the detrimental effect of singularities of the first kind is reduced by inserting a sufficiently high normalization parameter $R$ inside the transformation.

We want to comment further on two observations. First, we note that the performance of the waveguide saturates as $R$ is increased to about 50\% (see Supplemental Material, Sec. SIIIA [54]). This performance is far from its optimal value of $100 \%$, being degraded both by the limited validity of the thickness variation in small simulation domains (reducing the efficiency by $35 \%$; Fig. S2(a) [54]) and by the truncation itself (reducing the efficiency by 15\%; Fig. S2(b) [54]). Realistic coordinatebased designs inside larger simulation domains do not strain the validity of the thickness variation because the local planewave approximation is valid there (see Sec. IV). Effects due to truncations reduce as the normalization parameter is increased.

As a second observation, we are aware that larger values for $R$ also increase reflections at the input of the device. Indeed, for high values of $R$, the geometry no longer connects continuously to the untransformed region $\gamma=1$, so that waves refract [13]. This explains why the energy flows in Figs. 3(c) and 3(d) deviate slightly from the analytical ray trajectories in Fig. 3(a). Here, we focus on the actual performance close to the singularities inside the device. Problems related to the coupling of light into the waveguide may be addressed with 
appropriate couplers, e.g., adiabatic couplers that continuously increase the anisotropy of the core, which may also be designed with our technique [27].

We now generalize the above results for power maps $w=R z^{p}$ with an exponent $p \neq 2$. Close to a singularity, an arbitrary power map generates a radially dependent geometry component $\gamma(x, y)=p^{2} R^{2}\left(x^{2}+y^{2}\right)^{p-1}$. This geometry component leads to singularities of the first kind when $p>$ 1. As shown before, the effects due to these singularities may be softened by an appropriate normalization. From the dependence of the critical radius $r_{c} \approx\left(\frac{\gamma_{c}}{p^{2} R^{2}}\right)^{\frac{1}{2(p-1)}}$ on the normalization parameter $R$, we infer that the truncation will have more impact for $p$ values smaller than $\frac{3}{2}$. In the following section, we will consider the effects of singularities of the second kind $(p<1)$, which induce infinite anisotropies.

\section{A SCHWARZ-CHRISTOFFEL BEAM SPLITTER}

We now consider a Schwarz-Christoffel design of a beam splitter based on a sequence of power maps to demonstrate how we deal with singularities of the second kind (Fig. 4). The upper half of the $w$ plane is mapped onto a 6-polygon closing at infinity, with symmetric prevertices $a= \pm 1 / 2$ and $b= \pm 1$ and two outer prevertices at infinity [Fig. 4(a)]. For an arbitrary splitting angle $\alpha$, the Schwarz-Christoffel beam splitter is described by the following coordinate transformation (see Supplemental Material, Sec. SI [54]):

$$
R z(w)=C_{1} \int_{w_{0}}^{w} \frac{\left(w^{2}-a^{2}\right)^{\alpha / \pi}}{\left(w^{2}-b^{2}\right)^{\alpha / \pi}} d w+C_{2} .
$$

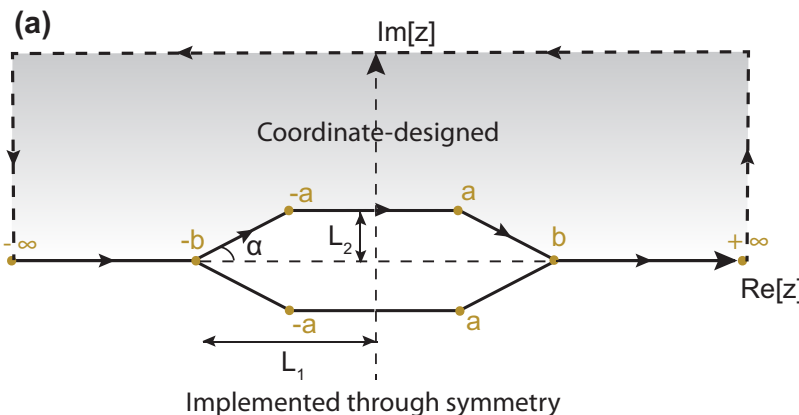

(c)

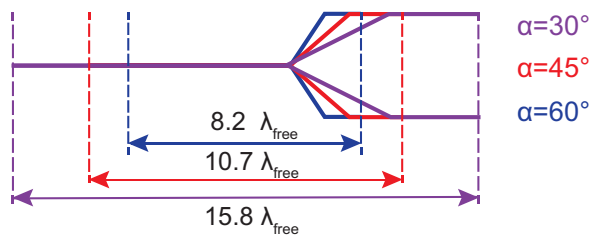

Note that we have again inserted a normalization parameter $R$ to deal with singularities of the first kind. We further chose the reference point $w_{0}$ to correspond to the initial prevertex $-b$, whose physical position is now exactly determined in the simulation domain by the integration constant $C_{2}$.

The beam-splitter design contains both kinds of singularities. Close to the prevertex $-b$, the transformation locally resembles a power map with $p=1+\frac{\alpha}{\pi}$, leading to a vanishing waveguide thickness (first kind), while close to the prevertex $-a$, the transformation resembles a power map with $p=1-$ $\frac{\alpha}{\pi}$, leading to infinite anisotropies (second kind). The resulting geometry (surface coloring) and thickness variation (floating surface) in Fig. 4(b) clearly contain singular signatures at the vertices $-b$ and $-a$, varying rapidly close to and reaching extremal values at the vertices.

We again use full-wave finite-element simulations to obtain the splitting efficiency, now for the Scharz-Christoffel beam splitter with an anisotropic core $\epsilon_{\perp} / \epsilon_{\|}=\gamma(x, y)$ and a thickness variation as in Eq. (1). These are determined by the integration of Eq. (6) (see Supplemental Material, Sec. SI [54]). Figure 4(d) shows the case of a beam splitter with a 30-degree splitting angle, having a very high efficiency (96\%). When the splitting angle increases from 30 to 60 degrees as in Fig. 4(c), however, the efficiency decreases from $96 \%$ to $86 \%$ (Figs. S3(a)-S3(c) [54]). Indeed, the singularity has more impact for higher angles $\alpha$ because the local power map deviates more strongly from 1 .

The previous simulations have already been optimized in terms of the normalization parameter $R$, so that singularities of the first kind could be truncated. For more information

(b)

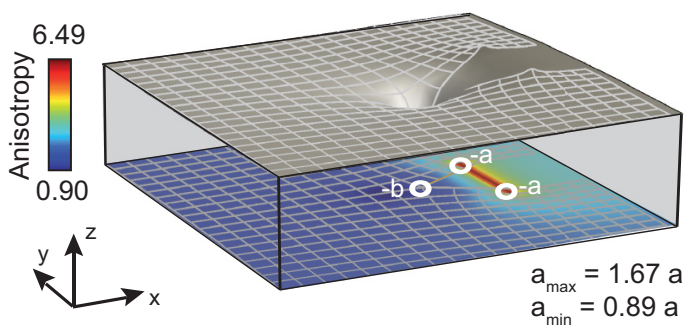

(d)

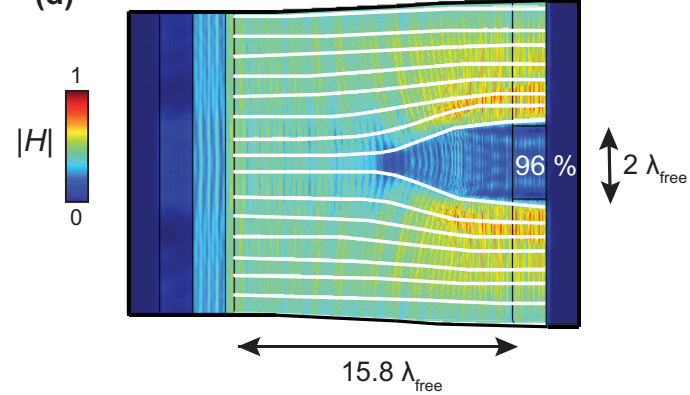

FIG. 4. A beam-splitter design based on a Schwarz-Christoffel transformation: (a) Sketch of the polygonal boundary corresponding to a beam splitter and/or interferometer. The coordinate transformation in the lower part of the beam splitter is obtained through symmetry. (b) The extremal values of the in-plane components of the geometry (surface coloring on the waveguide's symmetry plane) and the symmetric thickness variation (floating surface) clearly allow identification of the locations of the vertices corresponding to $-b$ and $-a$. (c) Identical separations may be obtained with different splitting angles $\alpha=30^{\circ}$ (purple), $\alpha=45^{\circ}$ (red), and $\alpha=60^{\circ}$ (blue) and splitting lengths. (d) The magnetic norm (surface coloring) and the power flow (white lines) behave as expected, splitting after the initial vertex with an efficiency $P_{\text {split }} / P_{\text {tot }}$ of $96 \%$ for a splitting angle of 30 degrees. 
on the performance of the splitter in terms of $R$, we refer to Sec. SIIIA of the Supplemental Material [54]. Here, we want to emphasize that normalizations cannot simultaneously soften singularities of the first and second kind. Indeed, the normalization parameters $R$ have to be larger than one to reduce the truncation disk of singularities of the first kind, whereas they have to be smaller than one to decrease the anisotropy at singularities of the second kind. Moreover, if the singularities at $-a$ are truncated, the guided waves do not feel the polygon vertex at $-a$ and go straight through (Figs. S3(e) and S3(f) [54]). Intuitively, the local wavelength at the singularity becomes very small so minor deviations from the initial coordinate-based design lead to severe deviations in the ray trajectories. Luckily, singularities of the second kind can be eliminated by inserting curve factors into the transformation.

\section{ELIMINATION OF SINGULARITIES WITH CURVE FACTORS}

Instead of introducing boundaries with sharp corners, as in Fig. 2, desired angular changes of the light trajectory can be induced by making use of the complex phase of analytic functions $C(w)$ on the upper $w$ plane,

$$
C(w)=w^{2}-\frac{1}{2}\left(a^{2}+b^{2}\right)+\sqrt{\left(w^{2}-a^{2}\right)\left(w^{2}-b^{2}\right)} .
$$

Figure 5(c) confirms that the phase of the curve factor $C(w)$ varies from 0 to $\pi$ along the edge $[-b,-a]$. Therefore, a power map $\alpha / \pi$ of the curve factor leads to an angular deviation $\alpha$ which is identical to that due to the singularity. We therefore modify Eq. (6) as follows:

$$
z(w)=\int_{w_{0}}^{w} \frac{C(w)^{\alpha / \pi}}{\left(w^{2}-b^{2}\right)^{\alpha / \pi}} d w+C_{2} .
$$

The curve factor does not induce any singularities on the real axis (it has a constant magnitude), uniquely defines the boundary (injective on $R$ ), and acts locally (the transformation approaches one at infinity). An extensive list of curve factors $C(w)$ can be found in Refs. [55,57] and were originally developed to fit transformed coordinate lines to any boundary. Note that in order to implement the transformation in a correct way, the Rieman surfaces of the curve factor should be accounted for as explained in Sec. SIC of the Supplemental Material [54].

Because the singularities at the prevertex $-a$ have been replaced by a smooth curve factor, the resulting anisotropy and thickness variation of the core are much more modest [Fig. 5(b)]. In particular, the anisotropy is half the value of the singular Schwarz-Christoffel design. The current design with curve factors imposes comparable splitting efficiencies of $96 \%$ for a splitting angle of $30^{\circ}$ [Fig. 5(d)] and these efficiencies are maintained for higher angles (Fig. S4 [54]). The price to pay is an increase in splitting length as the curve factor continuously redirects guided waves instead of deflecting them at a single point. (a)

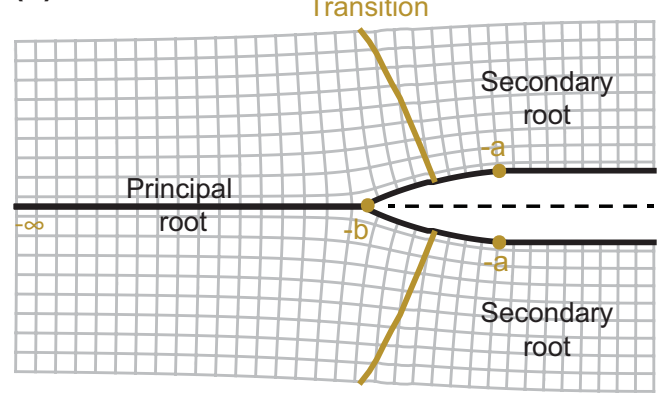

(c)

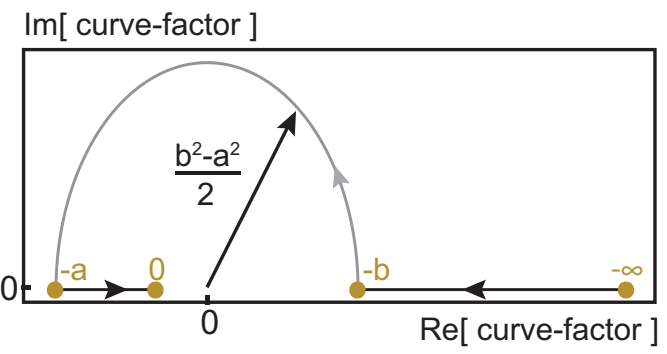

(b)

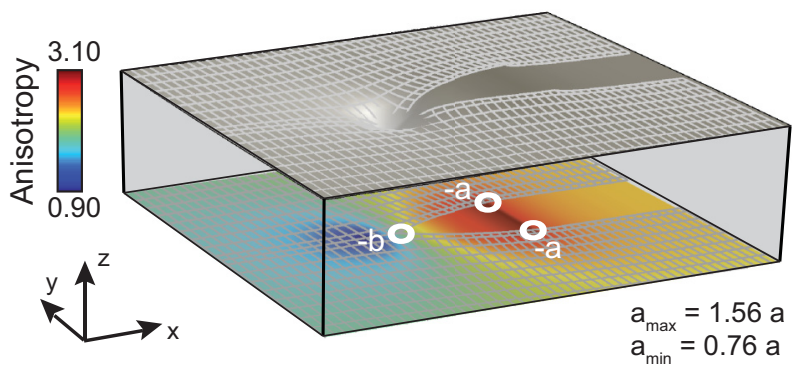

(d)

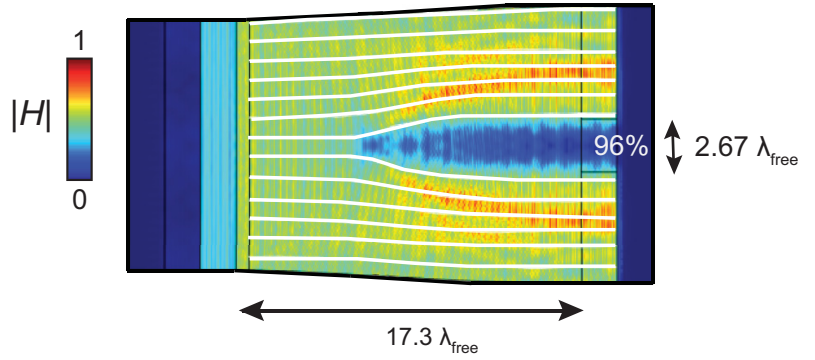

FIG. 5. A beam-splitter design with curve factors requires much more moderate optical parameters, i.e., the anisotropy is reduced by a factor of two. (a) The transformation generates a continuous geometry by making use of two Riemann surfaces, separated by the yellow line, corresponding to different signs of the square root in the curve factor. (b) The geometry (surface coloring on symmetry plane) and thickness variation of the upper interface (gray floating surface) occur in a more gradual and less extreme way than those of conventional Schwarz-Christoffel designs [Fig. 4], at the expense of a longer splitting length. (c) The curve factor induces a phase change from 0 to $\pi$ on the edge $[-b,-a]$ at a constant magnitude. (d) Full-wave simulations of the magnetic norm (surface coloring) and the power flow (white lines) demonstrate that the beam splitter splits with an efficiency of $96.4 \%$, comparable to the efficiency of the conventional Schwarz-Christoffel transformation. 


\section{CONCLUSION}

In this paper, we have numerically investigated how metamaterial waveguides based on Schwarz-Christoffel coordinate transformations perform in the presence of singularities that locally correspond to power laws $w=R z^{p}$ for $p>1$ and $p<1$. Our strategy for the elimination of optical singularities complements the approach in Ref. [42]. We soften or eliminate singularities while preserving the degree of isotropy of the initial design, whereas Ref. [42] transmutes singularities by adding anisotropy to the design.

Singularities of the first kind with $p>1$ are successfully softened by a normalization parameter $R$. For sufficiently high values of the normalization parameter, the coordinate transformation is implemented in an accurate way close to the singularity. This accurate implementation occurs at the expense of a mismatch between the transformed and untransformed waveguide at the input interface. Reflections due to this mismatch may be avoided by making use of suitable transformation-optical couplers, which can be designed with the technique in Ref. [27], or by introducing a spatially varying normalization parameter together with an appropriate boundary condition [43]. Quantitatively, we have demonstrated that the use of a sufficiently large normalization parameter may increase the efficiency by as much as $30 \%$ [Fig. 4(b)]. The actual improvement depends on the power of the local power map and is, therefore, device specific. Overall, the impact of a normalization parameter is highest when the exponent $p$ of the local power map is close to 1 .
Curve factors provide a second tool to replace singular power maps inside conformal coordinate transformations. In particular, curve factors induce the required angular changes along the wave's trajectory in a gradual way and do not rely on singularities. Our improved designs obtain much more moderate waveguide properties, e.g., the anisotropy of a beam splitter may be reduced twofold, which comes at the price of an increase in splitting length.

Because of the widespread use of Schwarz-Christoffel transformations in several branches of physics for reverse engineering and material designs, we anticipate that the removal of singularities in permittivity distributions with curve factors will prove to be a powerful technique for controlling waves along boundaries of any shape.

\section{ACKNOWLEDGMENTS}

S.V. and V.G. acknowledge the financial support of fellowships from the Research Foundation Flanders (FWOVlaanderen), Grants No. 11ZJ217N and No. 12O9115N. Work at Vrije Universiteit Brussel (VUB) was partially supported by the Research Council of the VUB and by the Interuniversity Attraction Poles programme of the Belgian Science Policy Office, under Grant No. IAP P7-35 "photonics@be”. Work at Chalmers University of Technology was supported by the COST Action No. MP1403 on Nanoscale Quantum Optics.
[1] H. Chen, C. Chan, and P. Sheng, Nat. Mater. 9, 387 (2010).

[2] M. Kadic, T. Bückmann, R. Schittny, and M. Wegener, Rep. Prog. Phys. 76, 126501 (2013).

[3] U. Leonhardt, Science 312, 1777 (2006).

[4] J. B. Pendry, D. S. Schurig, and D. R. Smith, Science 312, 1780 (2006).

[5] U. Leonhardt and T. Philbin, Geometry and Light: The Science of Invisibility (Dover, Mineola, NY, 2010).

[6] D. R. Smith, J. B. Pendry, and M. C. K. Wiltshire, Science 305, 788 (2004).

[7] J. B. Pendry, Nat. Mater. 5, 599 (2006).

[8] N. Engheta and R. W. Ziolkowski, Metamaterials: Physics and Engineering Explorations (Wiley, New York, 2006).

[9] N. M. Litchinitser and V. M. Shalaev, JOSA B 26, B161 (2009).

[10] C. M. Soukoulis and M. Wegener, Nat. Photon. 5, 523 (2011).

[11] S. Jahani and Z. Jacob, Nat. Nanotechnol. 11, 23 (2016).

[12] A. Greenleaf, M. Lassas, and G. Uhlmann, Physiol. Meas. 24, 413 (2003).

[13] M. Rahm, S. A. Cummer, D. Schurig, J. B. Pendry, and D. R. Smith, Phys. Rev. Lett. 100, 063903 (2008).

[14] L. Xu and H. Chen, Nat. Photon. 9, 15 (2015).

[15] V. Ginis, J. Danckaert, I. Veretennicoff, and P. Tassin, Phys. Rev. Lett. 113, 167402 (2014).

[16] J. Zhang, M. Wubs, P. Ginzburg, G. Wurtz, and A. V. Zayats, J. Opt. 18, 044029 (2016).

[17] M. Kraft, Y. Luo, and J. Pendry, Nano Lett. 16, 5156 (2016).

[18] R. Schittny, A. Niemeyer, M. Kadic, T. Bückmann, A. Naber, and M. Wegener, Opt. Lett. 40, 4202 (2015).

[19] F. Liu and J. Li, Phys. Rev. Lett. 114, 103902 (2015).
[20] A. I. Fernández-Domínguez, A. Wiener, F. J. García-Vidal, S. A. Maier, and J. B. Pendry, Phys. Rev. Lett. 108, 106802 (2012).

[21] M. Moccia, G. Castaldi, V. Galdi, A. Alù, and N. Engheta, Optica 3, 179 (2016).

[22] U. Leonhardt and T. G. Philbin, New J. Phys. 8, 247 (2006).

[23] V. Ginis, P. Tassin, B. Craps, and I. Veretennicoff, Opt. Express 18, 5350 (2010).

[24] M. McCall, A. Favaro, P. Kinsler, and A. Boardman, J. Opt. 13, 024003 (2010).

[25] M. Fridman, A. Farsi, Y. Okawachi, and A. Gaeta, Nature (London) 481, 62 (2012).

[26] J. M. Lukens, D. E. Leaird, and A. M. Weiner, Nature (London) 498, 205 (2013).

[27] S. Viaene, V. Ginis, J. Danckaert, and P. Tassin, Phys. Rev. B 93, 085429 (2016)

[28] Y. Liu, M. Mukhtar, Y. Ma, and C. K. Ong, JOSA A 30, 2280 (2013).

[29] P. Tassin, T. Koschny, M. Kafesaki, and C. M. Soukoulis, Nat. Photon. 6, 259 (2012).

[30] A. Boltasseva and H. Atwater, Science 331, 290 (2011).

[31] C. Soukoulis and M. Wegener, Science 330, 1633 (2010).

[32] Q. Zhao, J. Zhou, F. Zhang, and D. Lippens, Mater. Today 12, 60 (2009).

[33] O. Hess, J. Pendry, S. Maier, R. Oulton, J. Hamm, and K. Tsakmakidis, Nat. Mater. 11, 573 (2012).

[34] B. Wood, J. B. Pendry, and D. P. Tsai, Phys. Rev. B 74, 115116 (2006).

[35] Y. Huang, Y. Feng, and T. Jiang, Opt. Express 15, 11133 (2007). 
[36] A. Greenleaf, Y. Kurylev, M. Lassas, and G. Uhlmann, New J. Phys. 10, 115024 (2008).

[37] J. Li and J. B. Pendry, Phys. Rev. Lett. 101, 203901 (2008).

[38] N. Kundtz and D. Smith, Nat. Mater. 9, 129 (2010).

[39] J. Turpin, A. Massoud, Z. Jiang, P. Werner, and D. Werner, Opt. Express 18, 244 (2010).

[40] D. Schurig, J. Mock, B. Justice, S. Cummer, J. Pendry, A. Starr, and D. Smith, Science 314, 977 (2006).

[41] I. I. Smolyaninov, V. N. Smolyaninova, A. V. Kildishev, and V. M. Shalaev, Phys. Rev. Lett. 102, 213901 (2009).

[42] T. Tyc and U. Leonhardt, New J. Phys. 10, 115038 (2008).

[43] I. R. Hooper and T. G. Philbin, Opt. Express 21, 32313 (2013).

[44] S. A. R. Horsley, I. R. Hooper, R. C. Mitchell-Thomas, and O. Quevedo-Teruel, Sci. Rep. 4, 4876 (2014).

[45] Y. G. Ma, C. K. Ong, T. Tyc, and U. Leonhardt, Nat. Mater. 8, 639 (2009).

[46] Y. Liu, T. Zentgraf, G. Bartal, and X. Zhang, Nano Lett. 10, 1991 (2010).

[47] P. A. Huidobro, M. Nesterov, L. Martín-Moreno, and F. J. García-Vidal, Nano Lett. 10, 1985 (2010).

[48] M. Kadic, S. Guenneau, S. Enoch, P. A. Huidobro, L. Martín-Moreno, F. J. García-Vidal, J. Renger, and R. Quidant, Nanophotonics 1, 51 (2012).
[49] S. Xu, H. Xu, H. Gao, Y. Jiang, F. Yu, J. D. Joannopoulos, M. Soljacíc, H. Chen, H. Sun, and B. Zhang, Proc. Natl. Acad. Sci. USA 112, 7635 (2015).

[50] A. Vakil and N. Engheta, Science 332, 1291 (2011).

[51] P. A. Huidobro, M. Kraft, R. Kun, S. A. Maier, and J. B. Pendry, J. Opt. 18, 044024 (2016).

[52] L. Gabrielli, D. Liu, S. Johnson, and M. Lipson, Nat. Commun. 3, 1217 (2012).

[53] K. Yushin, L. Soo-Young, R. Jung-Wan, K. Inbo, H. Jae-Hyung, T. Heung-Sik, C. Muhan, and M. Bumki, Nat. Photon. 10, 647 (2016).

[54] See Supplemental Material at http://link.aps.org/supplemental/ 10.1103/PhysRevB.95.155412 for a discussion on why transmutation of singularities does not work, for methods to solve Schwarz-Christoffel transformations, and for technical information about the power map and beam splitters.

[55] J. Leathem, Philos. Trans. R. Soc. A 215, 439 (1915).

[56] R. Schinzinger and P. Laura, Conformal Mapping Methods and Applications (Courier Corporation, Mineola, NY, 2003).

[57] A. Andersson, J. Comput. Appl. Math. 233, 1117 (2009).

[58] S. Lang, Complex Analysis (Springer Science \& Business Media, New York, 2013), Vol. 103. 